\title{
Meniscus transplant: is still a step forward for good results in cartilage lesion progression and knee pain?
}

\author{
Dan G. Cojocaru', Gheorghe Bogdan Hogea ${ }^{1,2}$, Sorin Florescu, ${ }^{1,2}$, Jenel Marian Patrascu Jr. ${ }^{1,2}$, \\ Dragomir Halmagy ${ }^{1}$, Raymond Stoica ${ }^{1}$, Bogdan Anglitoiu ${ }^{1,2}$, Jenel Marian Patrascu ${ }^{1,2}$ \\ ${ }^{1}$ Emergency County Hospital, Timisoara, Romania \\ 2"Victor Babes" University of Medicine and Pharmacy, Timisoara, Romania
}

\begin{abstract}
Meniscus injuries are the most common traumatic conditions of the knee. If until the early 2000s, menisci were considered structures of no major importance in the knee joint, subsequent studies have shown their importance in the biomechanics of the knee. Their role is not only for the natural suspension of the joint, but also for the protection of the cartilage or the achievement of the efficient and uniform circulation of the synovial fluid. Therefore, disruption of this joint balance can lead, in the absence of a well-conducted treatment, to premature knee wear.

Most of the time, these meniscal tears are accompanied by associated lessions, ligaments or more severely cartilaginous lessions, creating a real challenge for the physician, specially for young pacients.

There are many conservative treatments of the damaged meniscus, imagined precisely in the idea of preserving the menisci as much as possible. However, this is not always possible, especially due to the type of traumatic injury.
\end{abstract}

Keywords: cartilage lession, meniscal tear, meniscectomy, meniscal transplant

\section{BACKGROUND}

With more than 700,000 arthroscopic meniscectomy per year only in USA alone (1), this kind of surgery it $\mathrm{s}$ become one of the most performed worldwide, not only in the ultra-specialized centers, But the most problematic and difficult to treat knee injury is represented by cartilage injury (2). This can occur as a result of a direct mechanism on the knee or through an indirect mechanism that increase the overload of certain territories in the load-bearing areas of the femoral condyles.

At this moment it is well known and proven by clinical studies that arthroscopic meniscectomies lead to premature wear of articular cartilage (3). This is due to the overload of the area where the torn portion of the meniscus has been removed. From a pathophysiological point of view, this oc- curs through the following mechanisms: increased pressure forces in the affected area, decreasing the vitality of the articular cartilage by diminishing its feeding capacity, both by the inefficiency of the synovial fluid circulation inside the joint and by decreasing its quality.

The nourishment and therefore the health of the articular cartilage is achieved by diffusion from the synovial fluid. It contains large amounts of glycosaminoglycans, prostaglandins, collagenases etc., has a structure and density that cannot be artificially reproduced (4). Its circulation inside the joint capsules and the achievement of efficient penetrability pressures on the cartilage is due to the disposition and biomechanics of the menisci. A larger or even smaller lesion disturbs the entire balance of the knee. Meniscectomies are even more important in the deterioration of this cycle, because smaller or larger portions of the meniscus 
are resected. Problems occur when large regions or even the entire meniscus is removed.

\section{MATERIALS AND METHODS}

This review article aims to update data in the evolution and results of both meniscal transplantation and knee osteoarthritis. After the year 2000 and until the end of 2018, the period in which this summary extends, the meniscal transplant had a permanent increase. This analysis aims is to evaluate this period. A simple search on the web with the words "meniscal transplant ostheoarthritis" gives us at least 1,300,000 results. Published studies that had the largest approach in the medical world were discussed. There are things that are unanimously accepted and common to all these studies.

\section{TYPES OF MENISCAL LESIONS}

There are many conservative treatments of the damaged meniscus, imagined precisely in the idea of preserving the menisci as much as possible. However, this is not always possible, especially due to the type of traumatic injury. In Figure 1 are reproduced the types of meniscal lesions.

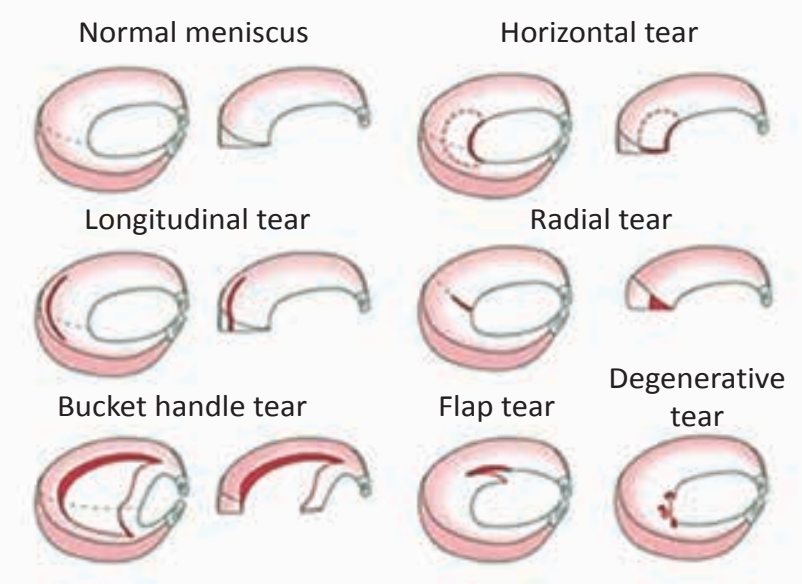

FIGURE 1. Meniscal lesions (Photo source: roadrunner.com)

Horizontal rupture affects the horizontal axis of the meniscus. Rupture "bucket handle" is an extended horizontal rupture and a portion of the meniscal body is broken and most often interposed between the bone planes, blocking the movement of full extension of the knee. Radial rupture is the most common type of rupture. It occurs in the area of the bearing surface, when a portion of the meniscus is crushed between the femoral condyle and the tibial plateau. Longitudinal rupture occurs in the longitudinal axis of the meniscus and most often affects its intensely vascularized position, having a high healing potential, both by conservative treatment (rest) and in surgical treatment (meniscal suture). Flap tear happens when a small area of the inner portion of the meniscus ruptures remaining attached to the meniscal body by a thin portion. Degenerative ruptures usually occur after the age of 50 and occur through prolonged wear, minor but repeated trauma, constant overload. It is expected that approximately $70 \%$ of the population over the age of 60 has a degenerative meniscus rupture (5). This type of rupture is often asymptomatic, but is often associated with an early degree of cartilage wear, creating with it a chronic inflammation in the knee that can lead to pain and joint discomfort. Surgery in this situation does not make the pain go away in the long run, but it is sometimes necessary to decongest the joint and prepare for conservative treatment of osteoarthritis (6).

In addition, a special importance is given by the area where this lesion occurs. There are 3 meniscal histological areas well known today: red zone, redwhite zone, white zone (see figure 2).

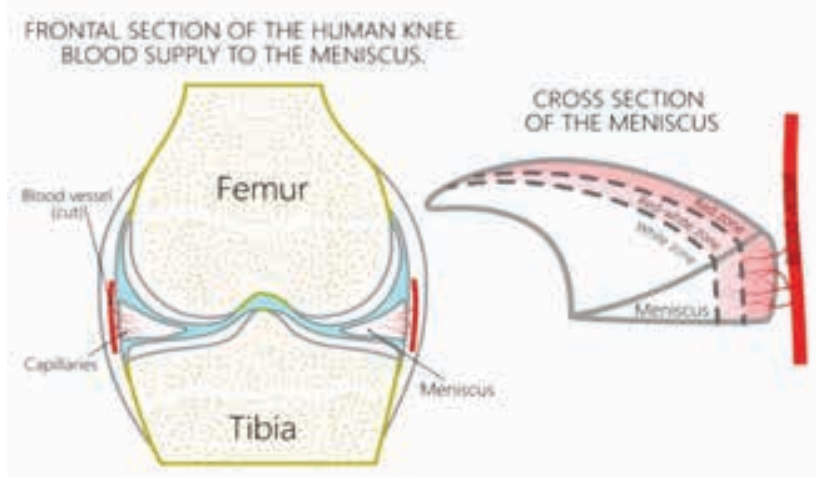

FIGURE 2. Blood supply of the meniscus by region (Photo source: roadrunner.com)

Depending on the location of the lesion, a meniscal suture can be tried: recommended for the red area, relatively recommended for the red-white area and not recommended when the lesion is in the white area (7).

But what happens when the entire meniscus can no longer be saved or a large area of its surface is excised? Studies show that the risk of cartilage injury occurring in the next 10 years is very high. The only option in this situation is the meniscal transplant.

\section{TYPES OF MENISCAL TRANSPLANT}

There are currently 2 major categories of meniscus transplantation and one under study: menis- 
cus transplant taken from a patient in clinical death (homotransplant - a); meniscus transplant of a synthetic biomaterial (artificial - b); meniscal transplant on individualized matrix of polyglycolic acid (in study, Research Department of Charité Hospital, Berlin - c) (see figure 3).
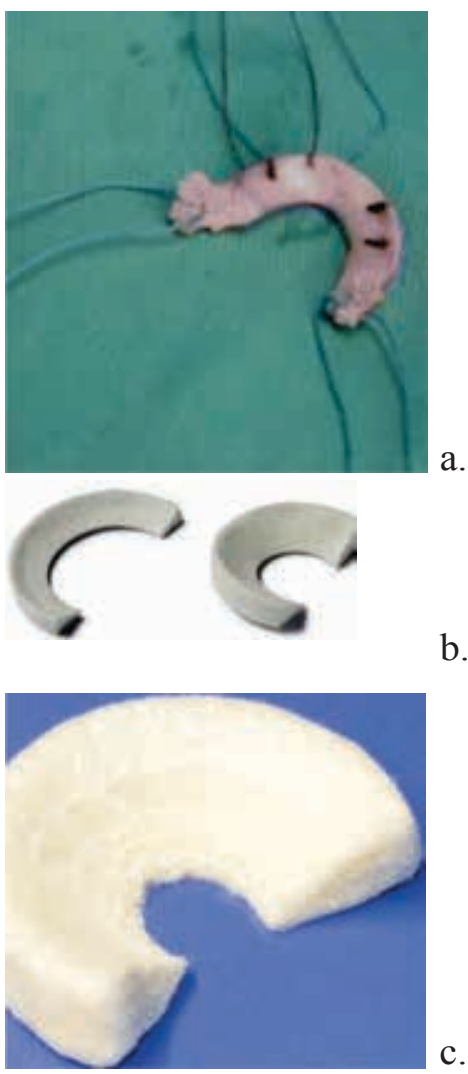

FIGURE 3 a, b, c. Types of meniscus transplant (7)

Knowing all these data, it is obvious the importance of the menisci both for the health of the articular cartilage and for the achievement of an efficient biomechanics of the whole joint. When one or more of the components of the knee joint do not work in normal parameters, the immediate tendency of the body is to compensate by its own mechanisms: synovial membrane hypertrophy, increased synovial fluid secretion, joint discomfort limiting joint overload (8).

With the appearance of cartilaginous lesions, their progression is almost inevitable. The meniscal transplant does not aim to cure these cartilage defects, but to slow down their evolution. Basically it can be considered as a "buying time" procedure for the joint (9). The mechanisms by joint function improves their condition after surgery are complex and must be carefully studied, but are based on regaining the anatomical biomechanics of the knee, the active participation of these new structures both in the uniform distribution of wear on the cartilage and in the synovial fluid circuit inside knee during periods of overload (10).

\section{MENISCAL TRANSPLANT OUTCOME}

Today there is no generally accepted concept for the benefit of meniscal transplantation. Many authors consider this technique beneficial in the sense of prolonging joint life (11), while others do not recommend it as a solution that brings remarkable benefits in terms of reducing pain or stopping the progression of arthrosis (12).

The analysis of conducted studies, especially after 2004, when this procedure was improved compared to the initial procedures dating back to the 90s, highlights the importance of meniscal transplantation in patients with extensive meniscectomy up to the age of 50 years, as a "buying time" procedure for the joint. The faster the joint transplant is performed, the better evolution for a long time (13).

Although there is progression of cartilage damage even after transplantation, there is an improvement of approximately $72 \%$ (14) in terms of quality of life, by decreasing pain, improving knee function and resuming sports activities.

Discussions are relatively controversial regarding patients with major meniscal defects and Outerbridge 4 injuries. Evaluation scores improved in only $20 \%$ of such patients, but the recommendation to perform a meniscal transplant remained the only viable option for them, especially if they are very young (15).

Today it is not possible to say which of the known methods of meniscal transplant is the most effective, both methods used having similar results. The encouragement also comes from the results obtained from the meniscal transplant experiments on a three-dimensional matrix of polyglycolic acid studies (16).

The best results are obtained when associated cartilaginous lesions are limited to grade II-III Outerbridge (17) and can be successfully performed both reconstructive procedures of articular cartilage and meniscus transplant, improving the result (18).

Meniscal transplantation improve joint function by regaining the anatomical biomechanics of the knee, but achieving it without a cooperative and preventive approach to the patient, leads to poor results (19). Weight control, proper lifestyle, joint gymnastics and maintaining muscle tone are also important steps in achieving a favorable longterm result (20).

\section{CONCLUSIONS}

Not being a usual procedure and requiring a thorough training in the field of arthroscopic sur- 
gery, meniscal transplantation obviously involves quite high costs. The question is whether it is worth these costs and how much a positive result is maintained in such a way that it is worth to be implemented in as many centers as possible.

Meniscal transplantation may be a viable and effective solution in the control of cartilaginous lesions and can result in improved joint function for specific pacients, specially the young one.

Today there is no general consensus and there are arguments both for and against this procedure.

Conflict of interest: none declared

Financial support: none declared

\section{REFERENCES}

1. Parkinson B, Smith N, Asplin L, Thompson P, Spalding T. Factors Predicting Meniscal Allograft Transplantation Failure. Orthop J Sports Med. 2016 Aug 19;4(8):2325967116663185.

2. Chalmers PN, Karas V, Sherman SL, Cole BJ. Return to high-level sport after meniscal allograft transplantation. Arthroscopy. 2013 Mar;29(3):539-44.

3. Lee BS, Bin SI, Kim JM, Kim WK, Choi JW. Survivorship After Meniscal Allograft Transplantation According to Articular Cartilage Status. Am J Sports Med. 2017 Apr;45(5):1095-1101.

4. Noyes FR, Barber-Westin SD. Meniscal Transplantation in Symptomatic Patients Under Fifty Years of Age: Survivorship Analysis. J Bone Joint Surg Am. 2015 Aug 5;97(15):1209-19.

5. Crook TB, Ardolino A, Williams LA, Barlow IW. Meniscal allograft transplantation: a review of the current literature. Ann R Coll Surg Engl. 2009 Jul;91(5):361-5.

6. Bloch B, Asplin L, Smith N, Thompson P, Spalding T. Higher survivorship following meniscal allograft transplantation in less worn knees justifies earlier referral for symptomatic patients: experience from 240 patients. Knee Surg Sports Traumatol Arthrosc. 2019 Jun;27(6):1891-1899.

7. van Arkel ER, de Boer HH. Human meniscal transplantation. Preliminary results at 2 to 5 -year follow-up. J Bone Joint Surg Br. $1995 \mathrm{Jul} ; 77(4): 589-95$.

8. Carr AJ, Price AJ, Glyn-Jones S, Rees JL. Advances in arthroscopyindications and therapeutic applications. Nat Rev Rheumatol. 2015 Feb;11(2):77-85.

9. Myers P, Tudor F. Meniscal allograft transplantation: how should we be doing it? A systematic review. Arthroscopy. 2015 May; 31(5):911-25

10. de Windt TS, Vonk LA, Brittberg M, Saris DB. Treatment and Prevention of (Early) Osteoarthritis Using Articular Cartilage Repair-Fact or Fiction? A Systematic Review. Cartilage. 2013 Jul;4(3 Suppl):5S-12S.

11. Smillie IS. The current pattern of internal derangements of the knee joint relative to the menisci. Clin Orthop Relat Res. 1967 Mar-Apr; 51:117-22.
Some authors consider it a useless procedure considering it as an intermediate stage until the moment of total knee arthroplasty (TKA), not justifying the high costs. On the other hand, the adepts of "buying time procedure" consider it to be in the advantage of the patient by prolonging the moment of TKA even if this means sometimes only a few years delay.

12. Katz JN, Losina E. Arthroscopic partial meniscectomy for degenerative tears: where do we stand? Osteoarthritis Cartilage. 2014 Nov;22(11):1749-51.

13. Stone AV, Loeser RF, Vanderman KS, Long DL, Clark SC, Ferguson CM. Pro-inflammatory stimulation of meniscus cells increases production of matrix metalloproteinases and additional catabolic factors involved in osteoarthritis pathogenesis. Osteoarthritis Cartilage. 2014 Feb;22(2):264-74.

14. Vundelinckx B, Vanlauwe J, Bellemans J. Long-term Subjective, Clinical, and Radiographic Outcome Evaluation of Meniscal Allograft Transplantation in the Knee. Am J Sports Med. 2014 Jul; 42(7):1592-9.

15. Rosso F, Bisicchia S, Bonasia DE, Amendola A. Meniscal allograft transplantation: a systematic review. Am J Sports Med. 2015 Apr;43(4):998-1007.

16. Moens K, Dhollander A, Moens P, Verdonk K, Verdonk R, Almqvist $\mathrm{KF}$, Victor J. Meniscal transplantation: still experimental surgery? A review. Acta Orthop Belg. 2014 Sep;80(3):403-13.

17. Zaffagnini S, Marcheggiani Muccioli GM, Lopomo N, Bruni D, Giordano G, Ravazzolo G, Molinari M, Marcacci M. Prospective long-term outcomes of the medial collagen meniscus implant versus partial medial meniscectomy: a minimum 10-year follow-up study. Am J Sports Med. 2011 May;39(5):977-85.

18. Van Der Straeten C, Byttebier P, Eeckhoudt A, Victor J. Meniscal Allograft Transplantation Does Not Prevent or Delay Progression of Knee Osteoarthritis. PLoS One. 2016 May 26;11(5):e0156183.

19. McDermott ID, Sharifi F, Bull AM, Gupte CM, Thomas RW, Amis AA. An anatomical study of meniscal allograft sizing. Knee Surg Sports Traumatol Arthrosc. 2004 Mar;12(2):130-5.

20. Roos H, Laurén M, Adalberth T, Roos EM, Jonsson K, Lohmander LS. Knee osteoarthritis after meniscectomy: prevalence of radiographic changes after twenty-one years, compared with matched controls. Arthritis Rheum. 1998 Apr;41(4):687-93. 